\title{
Post-traumatic Stress Disorder Symptoms in a Female Patient Following Repeated Teasing: Treatment with Gabapentin and Lamotrigine and the Possible Role of Sensitization
}

\author{
Akira Kishimoto', Yurie Goto', Kenji Hashimoto ${ }^{2}$ \\ ${ }^{1}$ Yonago Medical Corporation, Yonago Clinic, Yonago, ${ }^{2}$ Division of Clinical Neuroscience, Chiba University Center for Forensic Mental \\ Health, Chiba, Japan
}

\begin{abstract}
Post-traumatic stress disorder (PTSD) is a pathological response to trauma characterized by frequent recollections, recurrent nightmares, and flashbacks of the traumatic event(s). To date, the precise mechanisms underlying the development of PTSD remain unknown. Several studies have suggested that antiepileptic drugs, such as gabapentin and lamotrigine, may be effective in the treatment of PTSD symptoms. We report on a 15-year-old Japanese female junior high school student who developed PTSD symptoms following repeated teasing from male classmates. Additionally, we underscore the beneficial effects of treatment with gabapentin and lamotrigine on flashbacks and nightmares. This patient developed PTSD symptoms after repeated teasing from male classmates at school. Her flashbacks and nightmares were treated with a combination of gabapentin and lamotrigine. After recovery, treatment with lamotrigine alone controlled her symptoms. Our observations suggest that a process of sensitization may be involved in the development of PTSD symptoms. Additionally, gabapentin and/or lamotrigine were effective in the treatment of flashbacks and nightmares in this patient. Thus, doctors should consider using these anti-epileptic drugs as an alternative approach to treating PTSD symptoms.
\end{abstract}

KEY WORDS: Flashback; Gabapentin; Lamotrigine; Nightmare; Post-traumatic stress disorders; Sensitization.

\section{INTRODUCTION}

Post-traumatic stress disorder (PTSD) is a pathological response to trauma characterized by frequent recollections, recurrent nightmares, and images or flashbacks of the trauma(s). These symptoms are frequently associated with insomnia, irritability, impaired concentration, and increased vigilance. ${ }^{1)}$ The primary treatment options for people with PTSD are psychotherapy (e.g., exposure therapy, cognitive restricting, and stress inoculation training), medication, or a combination of both. The U.S. Food and Drug Administration has approved two medications, sertraline and paroxetine, for treating adults with PTSD. Additionally, other medications, including benzodiazepines, antipsychotics, and other antidepressants, have also been used as potential therapeutic drugs, although there is

\footnotetext{
Received: April 25, 2014 / Revised: June 21, 2014

Accepted: July 14, 2014

Address for correspondence: Akira Kishimoto, MD, PhD

Yonago Medical Corporation, Yonago Clinic, 80-1 Bakuro-machi 3-Chome, Yonago 683-0052, Japan

Tel: +81-859-34-1201, Fax: +81-859-35-5023

E-mail: ak-feb8@sea.chukai.ne.jp
}

little information about their effectiveness for PTSD. ${ }^{1)}$ Nonetheless, new therapeutic drugs for PTSD are needed, because the efficacy of current medications is limited.

Several clinical studies have suggested that the anti-epileptic drug, gabapentin, an analogue of $\gamma$-aminobutyric acid (GABA), may have beneficial effects on several PTSD symptoms. ${ }^{2-5)}$ Furthermore, a double-blind, placebo-controlled study showed that, compared with a placebo, another antiepileptic drug, lamotrigine, which functions as a voltage-sensitive sodium channel blocker, improved the re-experiencing and avoidance/numbing symptoms of patients with PTSD. ${ }^{6)}$ Whereas the precise mechanisms underlying PTSD symptoms remain unknown, these findings suggest that antiepileptic drugs, such as gabapentin and lamotrigine, may act as potential therapeutic drugs for this disorder. ${ }^{7,8)}$

Here, we describe the development of PTSD symptoms in a female junior high school student after repeated teasing from male classmates. Additionally, we also report on the beneficial effects of gabapentin and lamotrigine for controlling PTSD symptoms, such as nightmares and flashbacks.

(c) This is an Open-Access article distributed under the terms of the Creative Commons Attribution Non-Commercial License (http://creativecommons.org/licenses/by-nc/3.0) which permits unrestricted non-commercial use, distribution, and reproduction in any medium, provided the original work is properly cited. 


\section{CASE}

A15-year-old Japanese female patient was diagnosed with psychosomatic disorder (F-459), mixed anxiety and depressive disorder (F-41.2), and PTSD (F-43.1) according to International Classification of Diseases, 10th edition (ICD-10) criteria. During her first grade of junior high school, she was repeatedly teased by a male classmate, "S." When this teasing intensified, she received counseling at school and was then diagnosed with insomnia. She began to exhibit psychological and physical symptoms, such as stomach aches, headaches, and insomnia. At this point, she no longer felt able to attend school. She was then admitted to our clinic, reporting "I feel too heavyhearted to go to school," and "The school staff and school counselors do not listen to my complaints about the teasing." Additionally, she displayed psychotic symptoms such as, "I see the faces of the boys who teased me before go to bed," "I mistake night and day," and "Objects look distorted to me." At this time, she was not diagnosed with PTSD. Her score on the 17-item Hamilton Depression Rating Scale (HDRS) was 8, and she showed mild depressive symptoms and anxiety. She was treated with the hypnotic drug flunitrazepam $(2 \mathrm{mg})$ as well as an analgesic drug and she attended our clinic on a regular basis. However, the teasing continued. She reported "I fear their unbearable behavior toward me." She was unable to attend school due to her psychosomatic symptoms, which were brought on by the teasing. At this point, we decided to make a criminal report to the police. This news was broadcast on television and published in newspapers. One week later, she visited the clinic and reported "I have not been teased by 'S,' and I can go to school." At this time, she did not show depressive symptoms, and her score on the HDRS was 2.

Two weeks after returning to school, she was the target of intense teasing from student "A," a friend of "S." After 1-2 weeks, her vomiting, stomach aches, and headaches returned. This was followed by repeated nightmares and flashbacks. The flashbacks occurred between five and ten times per day, with a duration of approximately $30 \mathrm{~min}$. Additionally, she experienced physical symptoms, such as palpitations, trembling, vomiting, and fear and was unable to return to school. At this stage, she was diagnosed with PTSD, as she had four of the five symptoms needed for this diagnosis. She was started on gabapentin (400 mg). Her PTSD symptoms disappeared 3 days later, and she was able to return to school. Four months later, she agreed to be questioned by the police, and her flashbacks and nightmares re-emerged. The antiepileptic drug lamotrigine $(25 \mathrm{mg})$ was then added to her therapy, and the number of flashbacks decreased. After 2 weeks of treatment, the number of flashbacks and nightmares had been reduced by approximately $50 \%$. The dose of lamotrigine was therefore increased to $50 \mathrm{mg}$, and the incidence of flashbacks and nightmares began to decrease gradually, finally disappearing by the third week. After graduating from junior high school, she entered high school and enjoyed her school life. During this time, she was treated with flunitrazepam (2 mg), lamotrigine (50 mg), and fluvoxamine $(150 \mathrm{mg})$. Her encounters with students " $\mathrm{S}$ " and "A," who had teased her in junior high school, were not accompanied by flashbacks or nightmares. Therefore, gabapentin and fluvoxamine were discontinued. The remaining two drugs, lamotrigine and flunitrazepam, have been maintained due to her positive response. She is currently enjoying her life in high school and is free from flashbacks and nightmares.

Written informed consent for publication of this case report was obtained from the patient and her parent.

\section{DISCUSSION}

We report on a female student who developed PTSD symptoms after repeated teasing from male classmates at school. The precise mechanisms underlying the PTSD symptoms experienced by this patient are undefined. It is well known that exposure to traumatic stress is a requirement for the development of PTSD and that the amygdala plays a key role in fear and other emotional processes. ${ }^{9-11)}$ Furthermore, repeated exposure to the same stressor results in a progressive reduction in response magnitude, and this is thought to be mediated by structures such as the prefrontal cortex, paraventricular thalamus, and amygdala. However, exposure to a new stressor after either homotypic or heterotypic stressors causes an exaggerated response or sensitization, which may be mediated by enhanced activity in the basolateral amygdala, paraventricular thalamus, or locus coeruleus. ${ }^{12)}$ It has also been suggested that neuronal plasticity, such as amygdala kindling and sensitization, are models of PTSD. ${ }^{13)}$ Taken together, it is likely that this type of neuronal plasticity may have been involved in the developmental course of the PTSD symptoms experienced by this patient.

Here, we report a case showing the beneficial effects of gabapentin and lamotrigine on a female patient with PTSD. Inhibitory neurotransmission via GABA receptors 
in the amygdala is important for fear conditioning and, potentially, for regulating the process of fear learning. ${ }^{11)}$ It has also been reported that lamotrigine reduces GABAmediated neurotransmission in the rat amygdala, suggesting that the GABAergic system may play a role in its mechanism of action. ${ }^{14)}$ Although it is unclear how these two antiepileptic drugs, gabapentin and lamotrigine, alleviate PTSD symptoms, it seems that their modulation of GABA receptors may facilitate their actions in this regard. In this case, lamotrigine monotherapy was effective in the treatment of PTSD, as the removal of gabapentin did not lead to a worsening of symptoms in this patient. It has been reported recently that lamotrigine eliminated aggression in a patient with treatment-resistant PTSD. ${ }^{15)}$ This implies that lamotrigine may be useful for treating aggression and aggression-related symptoms in PTSD. Overall, it is highly likely that lamotrigine may be clinically effective in the treatment of PTSD, although further placebo-controlled, double-blind studies using large samples are necessary.

In conclusion, we documented the development of PTSD symptoms in a female subject who had experienced repeated teasing in school. Her clinical course implicates the operation of sensitization in the development of PTSD symptoms. Furthermore, this case suggests that the antiepileptic medications, gabapentin and lamotrigine, may be beneficial in the treatment of PTSD. Nonetheless, more detailed, randomized, double-blind studies should be performed to confirm the efficacy of gabapentin and lamotrigine for PTSD.

\section{REFERENCES}

1. National Institute of Mental Health. Post-traumatic stress disorder (PTSD). U.S. Department of Health and Human Services, National Institutes of Health. NIH Publication No. 08 6388. [cited 2014 Apr 1]. Available from Http: http:// www.nimh.nih.gov/health/topics/post-traumatic-stress-disorderptsd/index.shtml.
2. Berigan TR. Gabapentin and PTSD. J Clin Psychiatry 2002; 63:744.

3. Hamner MB, Brodrick PS, Labbate LA. Gabapentin in PTSD: a retrospective, clinical series of adjunctive therapy. Ann Clin Psychiatry 2001;13:141-146.

4. Malek-Ahmadi P. Gabapentin and posttraumatic stress disorder. Ann Pharmacother 2003;37:664-666.

5. Stein MB, Kerridge C, Dimsdale JE, Hoyt DB. Pharmacotherapy to prevent PTSD: Results from a randomized controlled proof-of-concept trial in physically injured patients. J Trauma Stress 2007;20:923-932.

6. Hertzberg MA, Butterfield MI, Feldman ME, Beckham JC, Sutherland SM, Connor KM, et al. A preliminary study of lamotrigine for the treatment of posttraumatic stress disorder. Biol Psychiatry 1999;45:1226-1229.

7. Aurora RN, Zak RS, Auerbach SH, Casey KR, Chowdhuri $\mathrm{S}$, Karippot A, et al; Standards of Practice Committee; American Academy of Sleep Medicine. Best practice guide for the treatment of nightmare disorder in adults. J Clin Sleep Med 2010;6:389-401.

8. Bajor LA, Ticlea AN, Osser DN. The Psychopharmacology Algorithm Project at the Harvard South Shore Program: an update on posttraumatic stress disorder. Harv Rev Psychiatry 2011;19:240-258.

9. Phelps EA, LeDoux JE. Contributions of the amygdala to emotion processing: from animal models to human behavior. Neuron 2005;48:175-187.

10. Yehuda R, LeDoux J. Response variation following trauma: a translational neuroscience approach to understanding PTSD. Neuron 2007;56:19-32.

11. Johansen JP, Cain CK, Ostroff LE, LeDoux JE. Molecular mechanisms of fear learning and memory. Cell 2011;147: 509-524.

12. Herman JP. Neural control of chronic stress adaptation. Front Behav Neurosci 2013;7:61.

13. Grillon C, Southwick SM, Charney DS. The psychobiological basis of posttraumatic stress disorder. Mol Psychiatry 1996;1:278-297.

14. Shiah IS, Yatham LN, Gau YC, Baker GB. Effect of lamotrigine on plasma GABA levels in healthy humans. Prog Neuropsychopharmacol Biol Psychiatry 2003;27:419423.

15. Kozarić-Kovačić D, Eterović M. Lamotrigine abolished aggression in a patient with treatment-resistant posttraumatic stress disorder. Clin Neuropharmacol 2013;36:94-95. 